\title{
Significant Correlations of SARS-CoV-2 Infection With Prevalence of Overweight/Obesity and Mean Body Mass Index in the SARS-CoV-2 Endemic Countries
}

\author{
Hidekatsu Yanai
}

\section{To the Editor}

A recent meta-analysis on the association between obesity and severe coronavirus disease 2019 (COVID-19) showed that COVID-19 patients with obesity were more severe and have a worse outcome than those without obesity (odds ratio (OR), 2.31; 95\% confidence interval (CI), 1.3 - 4.12) [1]. The metaanalysis on the association between obesity and COVID-19 mortality demonstrated that body mass index (BMI) $>25 \mathrm{~kg} /$ $\mathrm{m}^{2}$ was significantly associated with COVID-19 mortality (OR, 3.68; 95\% CI, 1.54 - 8.83; P < 0.003) [2]. Further, another meta-analysis showed that obesity was a significant risk factor for intensive care unit admission (OR, 1.21; 95\% CI, 1.002 1.46), as well as for invasive mechanical ventilation (OR, 2.05; 95\% CI, 1.16 - 3.64) in COVID-19 [3].

Very recently, I reported the potential risk factors for severe COVID-19 in obesity and the mechanisms for obesitymediated exacerbation of COVID-19 [4]. Briefly, angiotensin-converting enzyme (ACE) 2 is the cellular entry receptor of severe acute respiratory syndrome coronavirus 2 (SARSCoV-2) [5]. Increased ACE2 expression in overweight patients compared to those not overweight was observed [6], indicating that SARS-CoV-2 is more likely to enter into the human body in obese people as compared with non-obese people. Increased secretion of interleukin- 6 and tumor necrosis factor-alpha by accumulated adipose tissue induces insulin resistance, and results in the development of endothelial dysfunction and coagulopathy in obesity [7]. Elevation of inflammatory cytokines, endothelial dysfunction and procoagulant state already exist in obese people even before SARS-CoV-2 infection [4]. SARSCoV-2 infection may enhance elevation of inflammatory cytokines which leads to cytokine storm, and induces endothelial injury and thrombosis [4]. Further, the mechanical depression of ventilation due to morbid obesity may be associated with severity of COVID-19 [8].

Manuscript submitted September 1, 2020, accepted September 12, 2020

Published online November 2, 2020

Department of Diabetes, Endocrinology and Metabolism, National Center for Global Health and Medicine Kohnodai Hospital, 1-7-1 Kohnodai, Ichikawa, Chiba 272-8516, Japan. Email: dyanai@hospk.ncgm.go.jp

doi: https://doi.org/10.14740/cr1160
Recent meta-analyses suggest a significant association between obesity and severe COVID-19 and the underlying mechanisms have been elucidated, however, the association between overweight/obesity and the susceptibility to SARS$\mathrm{CoV}-2$ infection remains fully elucidated.

I studied the correlation of SARS-CoV-2 infection and COVID-19 mortality with prevalence of overweight/obesity and BMI in Top 50 SARS-CoV-2 endemic countries. The number of SARS-CoV-2 infected patients and deaths due to COVID-19 in each country was obtained by the report on August 27, 2020, by Johns Hopkins Coronavirus Resource Center. The prevalence of overweight (BMI $\geq 25 \mathrm{~kg} / \mathrm{m}^{2}$ ) and obesity $\left(\mathrm{BMI} \geq 30 \mathrm{~kg} / \mathrm{m}^{2}\right)$, and mean BMI were obtained by the 2016 report by the World Health Organization (WHO). The Top 50 SARS-CoV-2 endemic countries include, in order from the greatest number of SARS-CoV-2 infected patients, USA, Brazil, India, Russia, South Africa, Peru, Mexico, Colombia, Spain, Chile, Argentina, Iran, UK, Saudi Arabia, Bangladesh, Pakistan, France, Italy, Turkey, Germany, Iraq, Philippines, Indonesia, Canada, Qatar, Ukraine, Bolivia, Ecuador, Israel, Kazakhstan, Egypt, Dominican Republic, Panama, Sweden, China, Oman, Belgium, Kuwait, Romania, Belarus, Guatemala, Netherlands, UAE, Japan, Poland, Honduras, Singapore, Portugal, Morocco and Nigeria. I analyzed the correlation of the prevalence of overweight and obesity and mean BMI, with the number of SARS-CoV-2 infected patients and deaths due to COVID-19 per 100,000 people, by using the Spearman's correlation.

The prevalence of overweight was significantly and positively correlated with the number of SARS-CoV-2 infected patients and deaths due to COVID-19 (Fig. 1). The prevalence of obesity was significantly and positively correlated with the number of SARS-CoV-2 infected patients (Fig. 2). Mean BMI was significantly correlated with the number of SARS-CoV-2 infected patients and tended to be correlated with the number of deaths due to COVID-19 (Fig. 3).

Although enhanced expression of ACE2 in obese people may explain partly a significant association between obesity and the susceptibility to SARS-CoV-2 infection, the underlying precise mechanisms remain unknown. Further studies should be needed in the future.

In conclusion, my study showed a significant association between overweight/obesity and the susceptibility to SARS$\mathrm{CoV}-2$ infection by using the world-wide epidemiological data. 


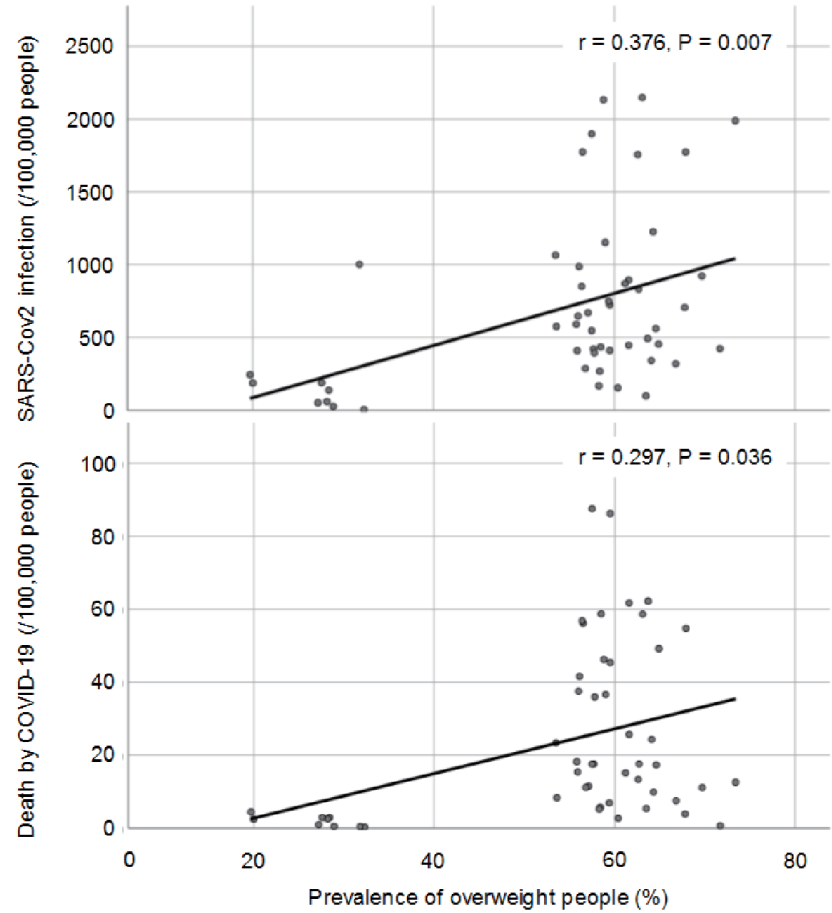

Figure 1. The correlation of prevalence of overweight with SARS-CoV-2 infection and deaths by COVID-19. SARS-CoV-2: severe acute respiratory syndrome coronavirus 2; COVID: coronavirus disease 2019.

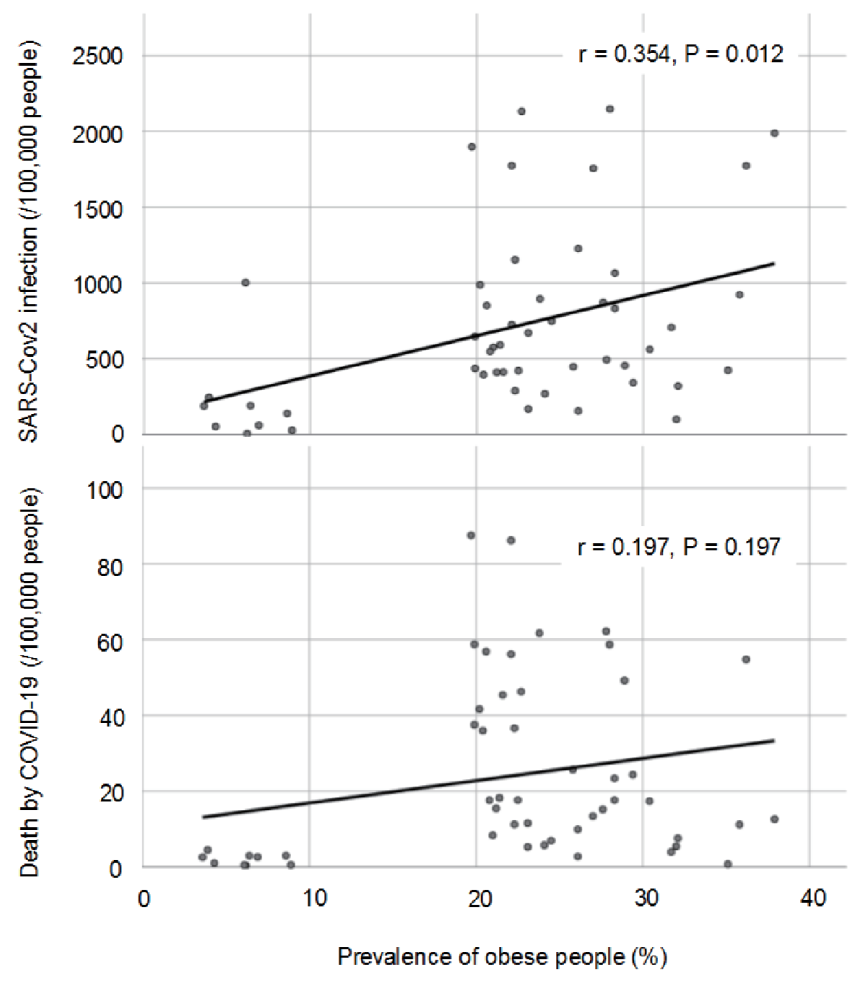

Figure 2. The correlation of prevalence of obesity with SARS-CoV-2 infection and deaths by COVID-19. SARS-CoV-2: severe acute respiratory syndrome coronavirus 2; COVID: coronavirus disease 2019.

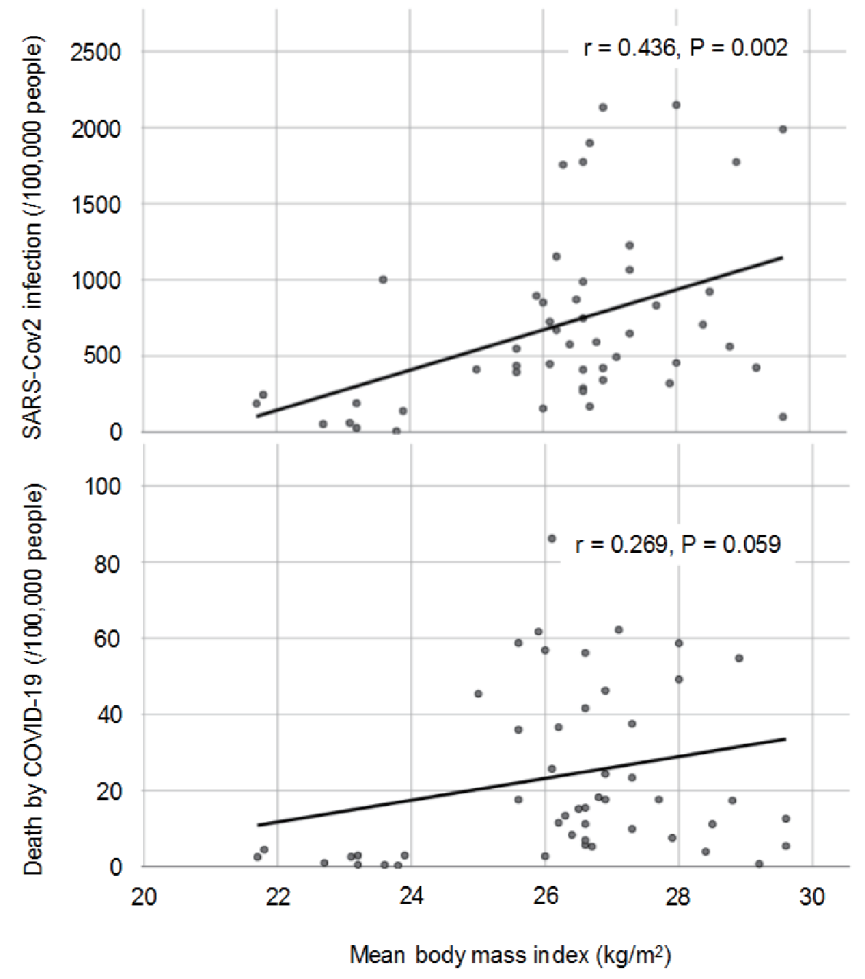

Figure 3. The correlation of mean body mass index with SARS-CoV-2 infection and deaths by COVID-19. SARS-CoV-2: severe acute respiratory syndrome coronavirus 2; COVID: coronavirus disease 2019.

\section{Acknowledgments}

I thank the staffs of the Division of Research Support, National Center for Global Health and Medicine Kohnodai Hospital.

\section{Financial Disclosure}

Author has no financial disclosures to report.

\section{Informed Consent}

Not applicable.

\section{Author Contributions}

H.Y. designed the research, and collected and analyzed data. H.Y. wrote and approved the final paper.

\section{Conflict of Interest}

The author declares that he has no conflict of interest concerning this article. 


\section{Data Availability}

The data supporting the findings of this study are available from the corresponding author upon reasonable request.

\section{References}

1. Yang J, Hu J, Zhu C. Obesity aggravates COVID-19: a systematic review and meta-analysis. J Med Virol. 2020.

2. Hussain A, Mahawar K, Xia Z, Yang W, El-Hasani S. Obesity and mortality of COVID-19. Meta-analysis. Obes Res Clin Pract. 2020;14(4):295-300.

3. Foldi M, Farkas N, Kiss S, Zadori N, Vancsa S, Szako L, Dembrovszky F, et al. Obesity is a risk factor for developing critical condition in COVID-19 patients: A systematic review and meta-analysis. Obes Rev. 2020;21(10):e13095.

4. Yanai H. Adiposity is the Crucial Enhancer of COVID-19. Cardiol Res. 2020;11(5):353-354.

5. Vaduganathan M, Vardeny O, Michel T, McMurray JJV, Pfeffer MA, Solomon SD. Renin-angiotensin-aldosterone system inhibitors in patients with COVID-19. N Engl J Med. 2020;382(17):1653-1659.

6. Higham A, Singh D. Increased ACE2 expression in bronchial epithelium of COPD patients who are overweight. Obesity (Silver Spring). 2020;28(9):1586-1589.

7. Yudkin JS. Abnormalities of coagulation and fibrinolysis in insulin resistance. Evidence for a common antecedent? Diabetes Care. 1999;22(Suppl 3):C25-30.

8. El-Solh A, Sikka P, Bozkanat E, Jaafar W, Davies J. Morbid obesity in the medical ICU. Chest. 2001;120(6):19891997. 